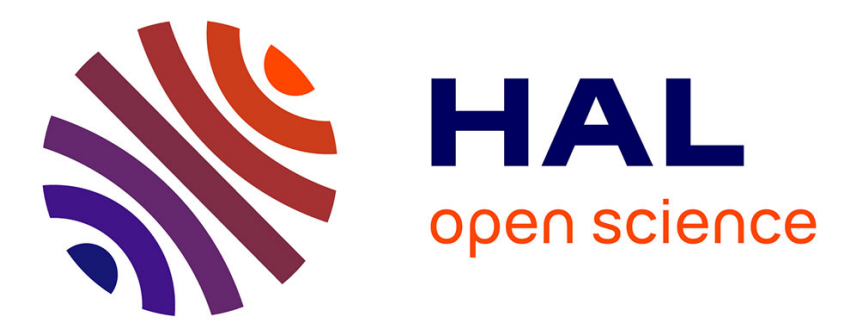

\title{
Variation du rendement de radioluminescence des scintillateurs organiques avec la perte d'énergie et le nombre de charges des particules ionisantes
}

\author{
J. Lopes da Silva, R. Voltz
}

\section{- To cite this version:}

J. Lopes da Silva, R. Voltz. Variation du rendement de radioluminescence des scintillateurs organiques avec la perte d'énergie et le nombre de charges des particules ionisantes. Revue de Physique Appliquée, 1972, 7 (2), pp.127-132. 10.1051/rphysap:0197200702012700 . jpa-00243603

HAL Id: jpa-00243603

https://hal.science/jpa-00243603

Submitted on 1 Jan 1972

HAL is a multi-disciplinary open access archive for the deposit and dissemination of scientific research documents, whether they are published or not. The documents may come from teaching and research institutions in France or abroad, or from public or private research centers.
L'archive ouverte pluridisciplinaire HAL, est destinée au dépôt et à la diffusion de documents scientifiques de niveau recherche, publiés ou non, émanant des établissements d'enseignement et de recherche français ou étrangers, des laboratoires publics ou privés. 
Classification

Physics abstracts

11.10

\title{
VARIATION DU RENDEMENT \\ DE RADIOLUMINESCENCE DES SCINTILLATEURS ORGANIQUES AVEC LA PERTE D'ÉNERGIE ET LE NOMBRE DE CHARGES DES PARTICULES IONISANTES
}

\author{
J. LOPES da SILVA $(*)$ et R. VOLTZ
}

Département de Physique des Rayonnements et d'Electronique Nucléaire, Centre de Recherches Nucléaires, Strasbourg, France

(Reçu le 27 novembre 1971, révisé le 6 mars 1972)

\begin{abstract}
Résumé. - On présente une étude expérimentale de l'influence de la nature des particules ionisantes sur le rendement de la radioluminescence, en distinguant la scintillation globale et ses deux composantes (prompte et différée). Un dispositif original permet de mesurer le nombre de photons émis, soit durant toute la scintillation, soit uniquement dans la composante différée, lorsqu'une particule chargée traverse une feuille mince de scintillateur plastique. On a utilisé une variété aussi grande que possible de rayonnements (électrons, protons, deutons, tritons, particules $\alpha$, ions ${ }^{3} \mathrm{He}^{++}$et azote), en accordant une attention particulière aux domaines d'énergie où le pouvoir ionisant est élevé. L'ensemble des résultats obtenus montre que les rendements de la scintillation et de ses deux composantes dépendent du nombre de charges et de la perte d'énergie spécifique des particules. Les données expérimentales sont en bon accord avec des expressions théoriques proposées récemment par les auteurs.
\end{abstract}

\begin{abstract}
An experimental study of the relative scintillation response of organic materials to different types of radiation is presented, considering separately the whole light emission and the two scintillation components. A device is described which allows one to mesure the number of emitted photons in the total scintillation and in the slow component, when a charged particle interacts with a thin scintillator foil. The different types of radiation used in this work were electrons (0.3-1 MeV), protons (0.3-4 MeV), deuterons $(0.3-1 \mathrm{MeV})$, tritons $(0.3-1 \mathrm{MeV}),{ }^{3} \mathrm{He}^{++}$ions (1-3 MeV), $\alpha$ particles (1-9 MeV) and nitrogen ions (10-80 MeV). The results show that the light yields for the scintillation and the two components depend on the charge number as well as on the specific energy loss of the particles; the data are in agreement with the theoretical expressions recently proposed by the authors.
\end{abstract}

Comme mesure du rendement de la scintillation, on utilise d'ordinaire, soit le nombre $\mathrm{d} S / \mathrm{d} E$ de photons émis par unité d'énergie perdue par la particule ionisante, soit le nombre $\mathrm{d} S / \mathrm{d} x$ de photons émis par unité de parcours de corpuscule chargé («luminescence spécifique ») [1], [2].

L'influence de la nature des rayonnements sur le rendement de la radioluminescence des scintillateurs organiques est généralement décrite par des relations semi-empiriques proposées respectivement par Birks [3] et Wright [4] :

$$
\begin{aligned}
& \frac{\mathrm{d} S}{\mathrm{~d} x}=A \frac{\mathrm{d} E}{\mathrm{~d} x}\left(1+B \frac{\mathrm{d} E}{\mathrm{~d} x}\right)^{-1}, \\
& \frac{\mathrm{d} S}{\mathrm{~d} x}=\frac{A}{2 B} \log \left(1+2 B \frac{\mathrm{d} E}{\mathrm{~d} x}\right),
\end{aligned}
$$

(*) Adresse actuelle : Núcleo de Química-Física Molecular Commissão de Estudos de Energia Nuclear (IAC). Instituto Superior Técnico. Lisboa - 1 Portugal. où $A$ et $B$ sont les constantes. Divers auteurs [1], [5]-[7] ont cependant souligné que l'ensemble des observations expérimentales publiées, notamment dans le cas de rayonnements de pouvoir ionisant élevé, ne peut être interprété par des relations de la forme (1).

De plus, l'expression de Birks ne tient pas compte de l'existence, dans la scintillation, de deux émissions dont l'origine physique est différente [2]. La première, désignée par «prompte » provient de la désexcitation radiative de molécules excitées dans le premier état singulet $S_{1}$, formées par des processus non radiatifs très rapides (conversion interne, recombinaison d'ions...) à partir des états primaires d'excitation et d'ionisation créés par les particules chargées; la décroissance de ce signal lumineux est exponentielle et identique à celle de la fluorescence. La deuxième émission, dite « différée », est due aux niveaux singulets $S_{1}$, résultant de l'interaction bimoléculaire d'états triplets dans. les zones de forte densité d'activation telles que les. traces des particules primaires et secondaires; dans. 
ce cas, le déclin est plus lent que pour le signal prompt, et a lieu suivant une loi non exponentielle, déjà étudiée en détail par ailleurs [2], [8], [9]. La luminescence spécifique globale $\mathrm{d} S / \mathrm{d} x$ doit donc être considérée comme la somme de deux termes $\mathrm{d} L / \mathrm{d} x$ et $\mathrm{d} L^{\prime} / \mathrm{d} x$ correspondant respectivement aux composantes prompte et différée, qu'il convient d'étudier séparément :

$$
\frac{\mathrm{d} S}{\mathrm{~d} x}=\frac{\mathrm{d} L}{\mathrm{~d} x}+\frac{\mathrm{d} L^{\prime}}{\mathrm{d} x}
$$

Nous avons repris l'examen des relations théoriques entre $\mathrm{d} L / \mathrm{d} x, \mathrm{~d} L^{\prime} / \mathrm{d} x$ et certaines quantités caractéristiques (nombre de charges $z$, perte d'énergie $\mathrm{d} E / \mathrm{d} x$ ) des particules nucléaires ; dans ce travail, déjà partiellement publié [2], [7], [10], nous avons distingué les états d'activation moléculaire produits dans la trace du corpuscule primaire, de ceux créés par les électrons secondaires énergiques; l'influence de la nature des rayonnements sur les luminescences spécifiques $\mathrm{d} L / \mathrm{d} x$ et $\mathrm{d} L^{\prime} / \mathrm{d} x$ est attribuée à une dégradation d'énergie électronique, résultant de l'interaction d'espèces activées voisines sur la trajectoire d'une même particule, primaire ou secondaire. On trouve ainsi, pour le signal prompt :

$$
\begin{array}{r}
\frac{\mathrm{d} L}{\mathrm{~d} x}=C\left\{\left(1-F_{\mathrm{s}}\right) \frac{\mathrm{d} E}{\mathrm{~d} x} \exp \left[-B_{\mathrm{s}}\left(1-F_{\mathrm{s}}\right) \frac{\mathrm{d} E}{\mathrm{~d} x}\right]+\right. \\
\left.+F_{\mathrm{s}} \frac{\mathrm{d} E}{\mathrm{~d} x}\right\},
\end{array}
$$

où $B_{\mathrm{s}}$ est une constante caractéristique des processus de dégradation de l'énergie des états singulets dans la trace des particules et $C$ une constante de proportionnalité ; $F_{\mathrm{s}}$ est une fonction de la quantité $z^{2}(\mathrm{~d} E / \mathrm{d} x)^{-1}$ et représente la proportion de l'énergie de la particule perdue en produisant des rayons $\delta$ d'énergie suffisante pour sortir de la trace. De même, pour le signal différé, on obtient l'expression :

$$
\frac{\mathrm{d} L^{\prime}}{\mathrm{d} x}=C^{\prime}\left[\Phi_{1} \frac{\mathrm{d} E}{\mathrm{~d} x} \exp \left(-B_{\mathrm{t}} \Phi_{1} \frac{\mathrm{d} E}{\mathrm{~d} x}\right)+\Phi_{2} \frac{\mathrm{d} E}{\mathrm{~d} x}\right]
$$

dans laquelle $B_{\mathrm{t}}$ caractérise les phénomènes de dégradation des états triplets dans la trace, et $C^{\prime}$ est une autre constante de proportionnalité ; $\Phi_{1} \mathrm{~d} E / \mathrm{d} x$ et $\Phi_{2} \mathrm{~d} E / \mathrm{d} x$ désignent respectivement les énergies, par unité de parcours du corpuscule incident, dissipées dans des zones de forte densité d'excitation par la particule primaire et les électrons secondaires rapides.

La validité de ces expressions théoriques a déjà été confirmée qualitativement par plusieurs études expérimentales [7], [10]-[14], mais les résultats sont encore trop fragmentaires pour permettre une comparaison précise aux relations (2) à (4); en particulier, toutes les mesures ont porté sur la scintillation globale sans chercher à la décomposer en ses deux composantes. Nous étions donc amenés à reprendre l'étude expérimentale du problème.
Dans ce but, nous avons mis au point un dispositif original permettant de mesurer directement une grandeur proportionnelle au nombre de photons émis, soit durant toute la scintillation, soit uniquement dans la composante lente de la radioluminescence, lorsqu'une particule nucléaire traverse une feuille mince de scintillateur plastique; les méthodes et dispositifs de mesure sont décrits dans une première partie de ce travail. Dans une deuxième partie, nous présentons les résultats de nos mesures, lesquelles ont porté sur une variété aussi grande que possible de particules (protons, deutons, tritons, particules $\alpha$, ions ${ }^{3} \mathrm{He}^{++}$, ions azote), en accordant une attention particulière aux domaines d'énergie où le pouvoir ionisant est élevé; de telles données sont en effet particulièrement intéressantes pour vérifier les prévisions théoriques sur l'influence du nombre de charges et de la perte d'énergie $\mathrm{d} E / \mathrm{d} x$ sur le rendement de la scintillation. La discussion des courbes expérimentales est abordée dans la dernière partie de cette étude ; elle est nécessairement brève dans le cadre de cette publication, consacrée surtout à l'aspect expérimental de notre travail.

1. Dispositif et méthodes de mesure. - Un film de scintillateur organique, d'épaisseur $\Delta x$ (de l'ordre du micron) est traversé par un corpuscule chargé d'énergie $E$; il s'agit de mesurer la perte d'énergie $\Delta E$ de la particule dans le scintillateur ainsi que les nombres de photons $\Delta S$ et $\Delta L^{\prime}$ émis respectivement dans la scintillation globale et dans le signal différé.

1.1 Principe (Fig. 1). - La particule ionisante, ayant traversé la feuille de scintillateur (F), tombe sur un détecteur à semiconducteur (DS). Celui-ci permet de déterminer la perte d'énergie à partir du déplacement du spectre d'amplitude des impulsions produites par la particule suivant qu'elle traverse ou non le scintillateur.

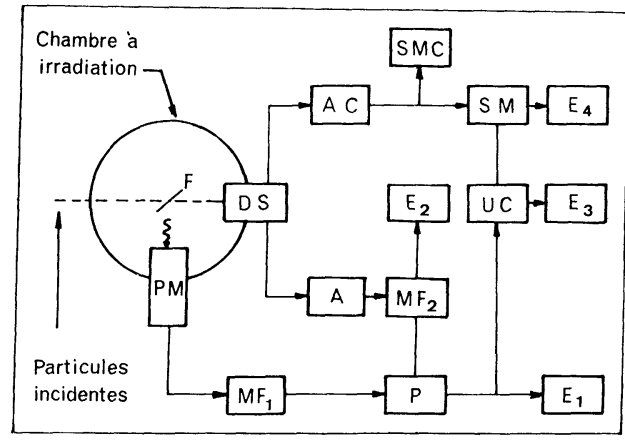

Fig. 1. - Schéma du montage expérimental.

Pour mesurer les faibles quantités de lumière émises lors du passage des particules à travers la feuille $\mathrm{F}$ on a employé un photomultiplicateur (PM) en compteur de photons [15]-[17]. Dans ce but, on règle l'ouverture d'un diaphragme placé devant le photo- 
multiplicateur afin que le nombre moyen $\bar{n}$ de photons tombant sur la cathode soit tel que $\bar{n} a \ll 1$, $a$ étant le rendement quantique de l'émission photoélectrique ; on montre que, dans ces conditions, le nombre par unité de temps, $N_{\mathrm{b}}$, d'impulsions anodiques du photomultiplicateur, est proportionnel à celui de photons arrivant sur la cathode. Si $\Delta I$ est l'intensité lumineuse émise par le film de scintillateur, on peut donc écrire que :

$$
N_{\mathrm{b}}=K \Delta I,
$$

où $K$ est un facteur de proportionnalité qui ne dépend que des conditions expérimentales, telles que la disposition géométrique et l'épaisseur de $\mathrm{F}$, ainsi que l'ouverture du diaphragme; si ces conditions sont fixées, le facteur $K$ reste constant pour toutes les mesures car la forme du spectre d'amplitude à photoélectron unique reste invariante pour tout le domaine spectral de la fluorescence du scintillateur, laquelle ne dépend pas de la nature du rayonnement ionisant. En pratique, pour réduire l'influence du bruit de fond du PM, on réalise des coïncidences entre les impulsions délivrées par le photomultiplicateur et celles issues du détecteur à semiconducteur. Les nombres, par unité de temps, de coincidences $\left(N^{\prime}\right)$, de signaux provenant du compteur DS $(N)$ et du PM $\left(N_{\mathrm{a}}\right)$ sont liés par la relation :

$$
N^{\prime}=2 \theta N_{\mathrm{a}} N,
$$

dans laquelle $\theta$ représente le temps de résolution du circuit de coïncidence et

$$
N_{\mathrm{a}}=N_{\mathrm{b}}+N_{\mathrm{c}},
$$

où $N_{\mathrm{c}}$ est le nombre d'impulsions de bruit de fond. D'après les relations (5) à (7), la quantité $\Delta I$ à mesurer est donc donnée par :

$$
\Delta I=\frac{1}{2 \theta K} \frac{N^{\prime}-N^{\prime \prime}}{N},
$$

$N^{\prime \prime}=2 \theta N N_{\mathrm{c}}$ étant le nombre de coïncidences fortuites, par unité de temps, en l'absence de scintillateur sur la trajectoire des ions.

1.2 DisPositiF EXPÉRIMENTAL. - La chambre à irradiation est représentée sur la figure 2. Elle est

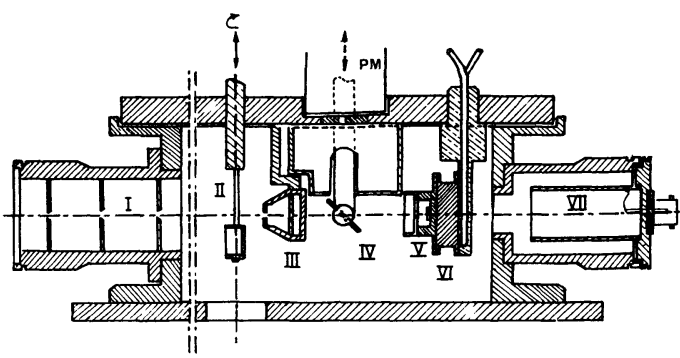

FIG. 2. - Chambre à irradiation; I et III : collimateurs ; II : support avec source radioactive, diaphragme et diffuseur métallique ; IV : scintillateur ; V : détecteur à semiconducteur ; VI : système de refroidissement (effet Peltier); VII : cylindre de Faraday. constituée d'une enceinte cylindrique en acier, raccordée à un ensemble de pompage et fermée par un couvercle qui peut tourner grâce à un roulement à billes. A l'intérieur de la chambre et solidaires du couvercle sont disposés une source radioactive, un diffuseur métallique, le scintillateur, le détecteur à semiconducteur et le photomultiplicateur. Le collimateur placé à l'entrée de l'enceinte permet de réduire éventuellement l'intensité d'un faisceau de particules accélérées. La disposition des différents éléments est telle que l'on peut, par rotation du couvercle, effectuer les mesures d'intensité de lumière et de perte d'énergie des particules dans les mêmes conditions géométriques, que l'irradiation du scintillateur ait lieu directement ou après diffusion des particules par la feuille métallique.

Le schéma du montage électronique est indiqué sur la figure 1. Le détecteur à semiconducteur (DS) est constitué par une diode à barrière de surface orsilicium ayant deux sorties isolées. La borne négative (or) est reliée à un amplificateur sensible à la charge (AC), qui délivre un signal d'amplitude proportionnelle à l'énergie de la particule détectée, lequel est analysé par un sélecteur multicanaux (SMC). La borne positive (silicium) de la diode est connectée à un amplificateur de tension (A) (*) suivi du circuit de mise en forme $\mathrm{MF}_{2}$, dont les signaux - de durée $(\approx 1,5 \mu \mathrm{s})$ supérieure à celle d'une scintillation commandent l'ouverture du circuit-porte P. Celui-ci permet d'effectuer les coïncidences entre les impulsions délivrées par le détecteur d'une part, et celles issues $\mathrm{du}$ photomultiplicateur PM (Dario $56 \mathrm{UVP} / 03$ ), puis mises en forme $\left(\mathrm{MF}_{1}\right)$, d'autre part.

$\mathrm{Au}$ cours de nos expériences, nous avons parfois travaillé avec des sources de particules non monocinétiques (particules $\alpha$ du dépôt actif du thorium, par exemple). Nous avons donc prévu, dans notre dispositif, une voie latérale destinée à effectuer une sélection en énergie : un sélecteur monocanal (SM), relié à l'amplificateur sensible à la charge, permet de n'appliquer que les signaux correspondant à une énergie déterminée au système de coïncidences $U C$, dont l'autre voie est reliée à la sortie du circuit-porte.

Les échelles $\mathrm{E}_{2}$ et $\mathrm{E}_{4}$ servent à compter respectivement le nombre global, par unité de temps, $N_{1}$, de particules détectées par la barrière de surface, et le nombre $N_{2}$ de corpuscules ayant l'énergie définie par le sélecteur monocanal. Avec les échelles $\mathrm{E}_{1}$ et $\mathrm{E}_{3}$, on mesure les nombres, $N_{1}^{\prime}$ et $N_{2}^{\prime}$, de coïncidences totales, ou ceux, $N_{1}^{\prime \prime}$ et $N_{2}^{\prime \prime}$, de coïncidences fortuites, ayant lieu respectivement au niveau des circuits $P$ et $\mathrm{UC}$.

(*) Cet amplificateur A est nécessaire, car en prélevant l'information à la sortie de (AC) (temps de montée $250 \mathrm{~ns}$ ), les fluctuations de l'instant de détection sont trop importantes et entraînent une incertitude temporelle pour l'ouverture du circuit-porte. 
1.3 Mesure Des QUANTITÉS DE LUMIÈRe $\Delta S$ ET $\Delta L^{\prime}$. - Le nombre des photons $\Delta S$ dans la scintillation totale est donné, d'après (8), par l'une ou l'autre des relations suivantes :

$$
\begin{aligned}
& \Delta S_{1}=\frac{1}{2 K \theta_{1}}\left(\frac{N_{1}^{\prime}-N_{1}^{\prime \prime}}{N_{1}}\right), \\
& \Delta S_{2}=\frac{1}{2 K \theta_{2}}\left(\frac{N_{2}^{\prime}-N_{2}^{\prime \prime}}{N_{2}}\right),
\end{aligned}
$$

où $\theta_{1}$ est la durée d'ouverture du circuit-porte, et $\theta_{2}$ le temps de résolution du circuit UC. Il faut noter que, pour un rayonnement monocinétique, on a nécessairement $\Delta S_{1}=\Delta S_{2}$; dans le cas contraire, la quantité $\Delta S$ est mesurée par $(9 b)$. Le facteur $K$ dans les expressions (9) dépend des conditions expérimentales mais non des caractéristiques des rayonnements incidents; pour que les valeurs de $\left.\left(N_{1}^{\prime}-N_{1}^{\prime \prime}\right) / N_{1}\right)$ ou de $\left(N_{2}^{\prime}-N_{2}^{\prime \prime}\right) / N_{2}$, obtenues pour différents types de particules, soient comparables, il faut donc que ces conditions (disposition géométrique du film, gain $\mathrm{du}$ photomultiplicateur, ...) soient maintenues constantes.

Pour mesurer le nombre de photons $\Delta L^{\prime}$ émis dans le signal différé, on retarde les impulsions issues du photomultiplicateur pendant un intervalle de temps $t^{\prime}$ supérieur à la durée du signal prompt. On a alors, dans la relation (5), $\Delta I=\Omega \Delta L^{\prime}, \Omega$ étant un facteur qui dépend de l'instant à partir duquel les photons " retardés » sont détectés. Par analogie avec ce qui précède, $\Delta L^{\prime}$ est donné par $(10 a)$ ou $(10 b)$ :

$$
\begin{aligned}
\Delta L_{1}^{\prime} & =\frac{1}{2 K \theta_{1} \Omega}\left(\frac{N_{1}^{\prime}-N_{1}^{\prime \prime}}{N_{1}}\right)_{\text {ret }}, \\
\Delta L^{\prime} & =\frac{1}{2 K \theta_{2} \Omega}\left(\frac{N_{2}^{\prime}-N_{2}^{\prime \prime}}{N_{2}}\right)_{\mathrm{ret}},
\end{aligned}
$$

avec $\Delta L^{\prime}=\Delta L_{1}^{\prime}=\Delta L_{2}^{\prime}$ si les particules incidentes sont monocinétiques, et $\Delta L^{\prime}=\Delta L_{2}^{\prime}\left(\neq \Delta L_{1}^{\prime}\right)$ dans le cas contraire. Notons que, comme la loi de décroissance du signal différé est indépendante de la nature et de l'énergie des rayonnements [2], [8], [9], il en est de même pour le facteur $\Omega$; les valeurs de $\Delta L^{\prime}$ mesurées pour différents types d'irradiation sont donc comparables.

2. Résultats expérimentaux. - Dans cette étude nous avons employé des protons $(0,3$ à $4 \mathrm{MeV})$, des deutons $(0,3$ à $1 \mathrm{MeV})$, des tritons $(0,3$ à $1 \mathrm{MeV})$, des ions ${ }^{3} \mathrm{He}^{++}(1$ à $3 \mathrm{MeV})$, des particules $\alpha(1$ à $9 \mathrm{MeV})$ et des ions azote $(10$ à $80 \mathrm{MeV})$. Les rayons $\alpha$ provenaient de sources radioactives $\left({ }^{210} \mathrm{Po}\right.$, dépôt actif de Th) ; les ions azote ont été accélérés par le cyclotron CEVIL (Orsay); dans ces deux cas l'énergie des particules a été modifiée à l'aide d'écrans métalliques d'épaisseurs appropriées. Les autres rayonnements ont été obtenus à l'aide d'accélérateurs de types van de Graaff (3 et $5 \mathrm{MeV}$ ) et Cockcroft-Walton (CRN, Strasbourg). A titre de comparaison, nous avons également fait des expériences avec des électrons de 0,3 à $1 \mathrm{MeV}$ (sources de ${ }^{113} \mathrm{In},{ }^{137} \mathrm{Ba}$ et ${ }^{207} \mathrm{~Pb}$ ) dont la perte d'énergie $\mathrm{d} E / \mathrm{d} x$ est beaucoup plus faible que pour les ions lourds.

Le scintillateur plastique utilisé (NE $102 \mathrm{~A}$ : polyvinyltoluène contenant des solutés fluorescents) a été préparé sous forme de films minces suivant un mode analogue à celui décrit par Green [18]. L'épaisseur $\Delta x$ des feuilles de plastique était de l'ordre de $1 \mu$ pour que l'énergie $\Delta E$ dissipée par chaque particule soit très inférieure à son énergie $E(\Delta E / E \ll 1)\left({ }^{*}\right)$; dans ces conditions, les quantités mesurées $\Delta E, \Delta S, \Delta L^{\prime}$ et $\Delta x$ peuvent être assimilées aux quantités différentielles $\mathrm{d} E, \mathrm{~d} S, \mathrm{~d} L^{\prime}$ et $\mathrm{d} x$ qui figurent dans les relations théoriques à vérifier.

\subsection{Perte D'éNergie des Particules et épaisseur} DES FEUILLES. - Les résultats expérimentaux sur la perte d'énergie $\Delta E$ des particules dans le film de scintillateur, par comparaison aux données calculées de $\mathrm{d} E / \mathrm{d} x$, permettent de déterminer avec précision l'épaisseur $\Delta x$ de la feuille organique.

Sur les figures 3 et 4 , nous avons représenté en coordonnées logarithmiques, les valeurs mesurées de $\Delta E$ en fonction de l'énergie $E$ de protons $(0,3$ à $3 \mathrm{MeV})$, d'ions ${ }^{3} \mathrm{He}^{++}(1$ à $3 \mathrm{MeV}),{ }^{4} \mathrm{He}^{++}(1$ à $9 \mathrm{MeV})$

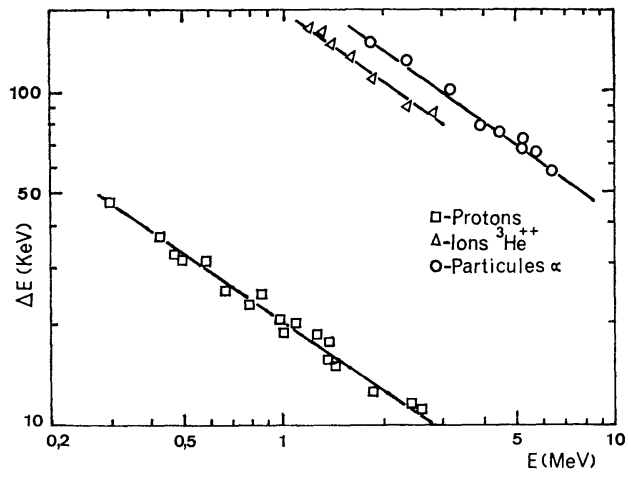

Fig. 3. - Perte d'énergie de protons, ions ${ }^{3} \mathrm{He}^{++}$et particules $\alpha$ dans un film de scintillateur organique : points expérimentaux et courbes calculées.

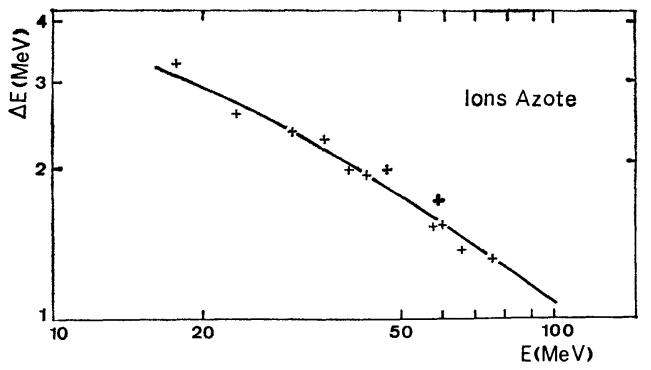

Fig. 4. - Perte d'énergie d'ions azote dans un film de scintillateur plastique : points expérimentaux et courbes calculées.

$\left({ }^{*}\right)$ A titre d'exemples, indiquons que, pour avoir une perte d'énergie $\Delta E$, telle que $\Delta E / E=0,1$, il faut $\Delta x=1 \mu$ pour un deuton de $0,2 \mathrm{MeV}$ et un ion azote de $10 \mathrm{MeV}$. 
et azote $(15$ à $80 \mathrm{MeV})$, pour un scintillateur d'épaisseur donnée.

Si les épaisseurs $\Delta x$ sont assez faibles pour que $\Delta E / E \ll 1$, les résultats des mesures de $\Delta E$ doivent être tels que :

$$
\Delta E=\frac{\mathrm{d} E}{\mathrm{~d} x} \Delta x
$$

où $\mathrm{d} E / \mathrm{d} x$ peut être calculé à partir des expressions de Bethe

$$
\frac{\mathrm{d} E}{\mathrm{~d} x}=\frac{2 \pi e^{4}}{m E} z^{2} N Z \log \frac{2 m E}{M I},
$$

( $e$ et $m$ : charge et masse électroniques; $z$ et $M$ : nombre de charges et masse du corpuscule chargé). $N Z$ représente la densité électronique, et a été évaluée connaissant la composition $\left(\mathrm{CH}_{1,105}\right)$ et la densité $\left(1,032 \mathrm{~g} . \mathrm{cm}^{-3}\right) \mathrm{du}$ scintillateur :

$$
N Z=3,36 \times 10^{23} \text { électrons par } \mathrm{cm}^{3} .
$$

$I$ est le potentiel d'ionisation moyen; il a été déterminé suivant les indications de Sternheimer en utilisant la règle d'addition de Bragg [19], [22]. Dans le cas particulier des ions azote, nous avons tenu compte des valeurs de la charge effective, données par Northcliffe [20]. Dans ces conditions, on vérifie que la relation (11) est satisfaite dans tous les cas, en prenant une même valeur pour l'épaisseur $\Delta x$ : c'est ainsi que les courbes en traits pleins sur les figures 3 et 4 représentent les quantités $(\mathrm{d} E / \mathrm{d} x) \Delta x$, calculées avec $\Delta x=4,9 \times 10^{-5} \mathrm{~cm}$. Il faut noter que la précision de cette valeur numérique dépend de celle de l'énergie $I$; elle souffre donc des limites de la validité de la règle de Bragg mises en évidence récemment dans des cas de milieux organiques solides [23]. Les valeurs ainsi obtenues pour l'épaisseur $\Delta x$ peuvent cependant être considérées comme plus précises que celles déterminées par pesée (méthode particulièrement imprécise, car le poids du film est très inférieur à celui de son support).

2.2 LUMINESCENCE SPÉCIFIQUE. - Connaissant l'épaisseur $\Delta x$ de la feuille de scintillateur, ayant déterminé les quantités de lumières $\Delta S$ et $\Delta L^{\prime}$ d'après les méthodes du paragraphe 1.3 , on a des mesures des quantités $\Delta S / \Delta x$ et $\Delta L^{\prime} / \Delta x$, données par :

$$
\begin{aligned}
& \frac{1}{\Delta x} \frac{N^{\prime}-N^{\prime \prime}}{N}=2 K \theta \frac{\Delta S}{\Delta x}, \\
& \frac{1}{\Delta x}\left(\frac{N^{\prime}-N^{\prime \prime}}{N}\right)_{\text {ret }}=2 K \theta \Omega \frac{\Delta L^{\prime}}{\Delta x} .
\end{aligned}
$$

En pratique, les résultats concernant les corpuscules de charges différentes ne sont pas nécessairement comparables, les facteurs $K$ étant caractéristiques de conditions expérimentales éventuellement non identiques; il fallait donc procéder à une normalisation, laquelle a été réalisée en prenant comme référence les données obtenues avec des particules $\alpha$ de ${ }^{210} \mathrm{Po}$; pour évaluer le facteur de normalisation, on a, après avoir déterminé les quantités (13) et (14) pour un type de rayonnement donné, effectué ces mêmes mesures avec les rayons $\alpha$, en conservant identiques tous les paramètres expérimentaux, ce qui est possible avec notre chambre à irradiation.

Les différentes données expérimentales étant ainsi comparables, sont indiquées sur la figure 5 pour les

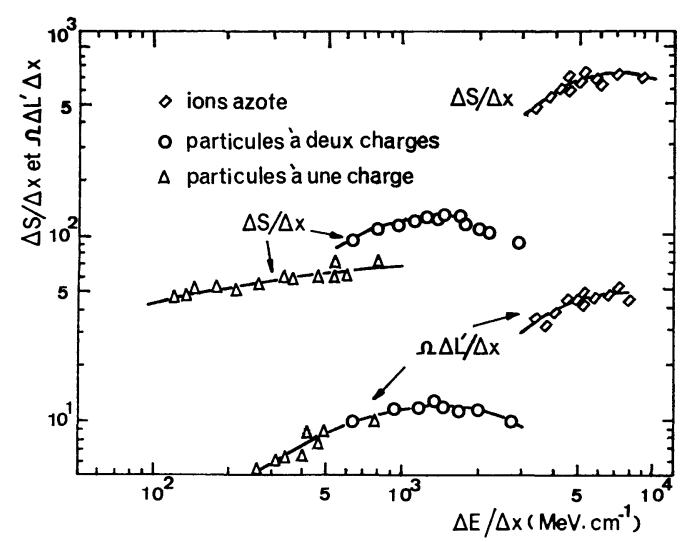

FIG. 5. - Résultats expérimentaux sur les variations des luminescences spécifiques $\Delta S / \Delta x$ et $\Omega \Delta L^{\prime} / \Delta x$ en fonction du pouvoir d'arrêt d'ions lourds (résultats normalisés en prenant comme unité de luminescence spécifique celle correspondant aux électrons de conversion ${ }^{137} \mathrm{Ba}$ ).

particules lourdes chargées; l'échelle de luminescence spécifique a été choisie en prenant comme unité celle correspondant aux électrons de conversion de ${ }^{137} \mathrm{Ba}$; on note, par exemple, que les particules $\alpha$ induisent une luminescence spécifique $\Delta S / \Delta x$ égale à environ 100 fois celle provoquée par un électron de $0,624 \mathrm{MeV}$ $\left({ }^{137} \mathrm{Ba}\right)$, ce qui est en accord avec les observations d'autres auteurs [1]. Sur la figure 6, nous avons représenté les résultats de nos mesures avec les électrons ; dans ce cas, nous n'avons pu étudier l'émission différée qui était trop faible pour que l'on puisse distinguer nettement les nombres $N^{\prime}$ et $N^{\prime \prime}$ qui interviennent dans les relations (10).

3. Discussion. - L'ensemble de nos résultats expérimentaux montre que les rendements de la scintillation et de ses deux composantes dépendent du nombre de charges $z$ et de la perte d'énergie spécifique $\mathrm{d} E / \mathrm{d} x$ des particules incidentes.

3.1 INFLUENCE DU NOMBRE DE CHARges. - Elle n'est pas prévue par les relations (1) ; dans les expressions (3) et (4), par contre, les quantités $F_{\mathrm{s}}, \Phi_{1}$ et $\Phi_{2}$ dépendent de la variable $z^{2}(\mathrm{~d} E / \mathrm{d} x)^{-1}$ : à pouvoir d'arrêt égal, des corpuscules de masse différente, mais de même charge, doivent provoquer l'émission de la même quantité de lumière dans la scintillation globale, comme dans la composante différée. Ceci est bien en accord avec les observations que nous avons faites avec les ions de charges +1 et +2 (Fig. 5). 
Nos relations théoriques prévoient, par ailleurs, que pour une valeur donnée de la variable $\mathrm{d} E / \mathrm{d} x$, la particule la plus chargée induit les luminescences spécifiques $\mathrm{d} S / \mathrm{d} x$ et $\mathrm{d} L^{\prime} / \mathrm{d} x$ les plus élevées ; l'examen des résultats expérimentaux de la figure 5 montre qu'il en est bien ainsi : avec les rayons $\alpha$ et les protons, caractérisés par une perte d'énergie

$$
\mathrm{d} E / \mathrm{d} x \approx 700 \mathrm{MeV} \cdot \mathrm{cm}^{-1}
$$

par exemple, le rapport des valeurs mesurées de la quantité $\mathrm{d} S / \mathrm{d} x$ est de l'ordre de 1,5 ; ce rapport est encore plus grand dans le cas des ions azote et des particules $\alpha$ de même perte d'énergie spécifique, puisqu'il est d'environ 5 pour $\mathrm{d} E / \mathrm{d} x \approx 3000 \mathrm{MeV} \cdot \mathrm{cm}^{-1}$. Notons aussi que, si les données expérimentales relatives à la luminescence du signal différé, déterminées avec des protons et des particules $\alpha$ de pouvoirs ionisants égaux, sont du même ordre de grandeur, il n'en est pas de même pour les ions azote avec lesquels on obtient des valeurs de $\Delta L^{\prime} / \Delta x$ nettement supérieures : on voit, par exemple, que pour

$$
\mathrm{d} E / \mathrm{d} x \approx 3000 \mathrm{MeV} . \mathrm{cm}^{-1},
$$

ces derniers corpuscules provoquent une luminescence différée égale à 3 fois celle mesurée avec des rayons $\alpha$ de même perte d'énergie.

3.2 INFLUENCE DU POUVOIR D'ARRÊT. - Pour des valeurs croissantes de $\mathrm{d} E / \mathrm{d} x$, la relation $(1 b)$ de Wright prévoit une augmentation continue de $\mathrm{d} S / \mathrm{d} x$ et celle $(1 a)$ de Birks une croissance asymptotique vers une valeur constante; d'après les relations (2) à (4) que nous proposons, par contre, les quantités $\mathrm{d} S / \mathrm{d} x$ et $\mathrm{d} L^{\prime} / \mathrm{d} x$ doivent passer par un maximum et décroître pour des pouvoirs d'arrêt élevés. La figure 5 montre que cette prévision est bien vérifiée dans le cas des particules de charge +2 . L'existence d'un tel maximum a également été mise en évidence par d'autres auteurs [6], [21] en mesurant la luminescence spécifique $\mathrm{d} S / \mathrm{d} x$ d'un cristal d'anthracène irradié par des rayons $\alpha$. Dans les cas des corpuscules à une charge et des ions azote, nous n'avons pu conclure à l'existence d'un maximum, car nous aurions dû réaliser des expériences dans des domaines d'énergies trop faibles pour que la définition en énergie reste satisfaisante.

Notons enfin que, d'après toutes les relations mentionnées dans l'introduction, la luminescence spécifique $\mathrm{d} S / \mathrm{d} x$ est une fonction linéaire du pouvoir d'arrêt $\mathrm{d} E / \mathrm{d} x$ si celui-ci est suffisamment faible; tel est le cas pour les électrons que nous avons utilisés dans nos expériences, comme le montre la figure 6 .

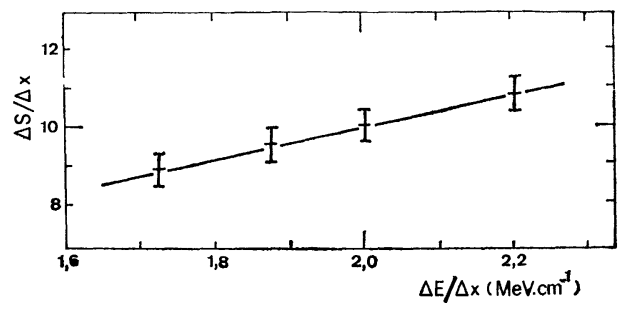

Fig. 6. - Luminescence spécifique $\Delta S / \Delta x$ en fonction de la perte d'énergie $\Delta E / \Delta x$ d'électrons.

L'interprétation théorique détaillée des courbes expérimentales présentées dans ce travail, fera l'objet d'une publication ultérieure, dans laquelle nous insisterons, en particulier, sur les indications nouvelles que fournissent nos résultats sur les effets primaires des rayonnements dans les milieux organiques.

Remerciements. - L'un de nous (J. Lopes da Silva) remercie l'Instituto de Alta Cultura pour l'autorisation d'absence provisoire de la Commissão de Estudos de Energia Nuclear, Lisboa, ainsi que la Fundação Calouste Gulbenkian pour la bourse qu'elle lui a accordée. Nous remercions également M. J. Miehé pour l'aide qu'il nous a apportée.

\section{Bibliographie}

[1] Birks (J. B.), The Theory and Practice of Scintillation Counting, Pergamon Press, 1964, Chap. 7.

[2] Voltz (R.), in « Actions chimiques et biologiques des radiations » (M. Haïssinsky éd.), Masson, Paris, 1969, 13, 1.

[3] Birks (J. B.), Proc. Phys. Soc., 1950, A 63, 1294.

[4] Wright (G. T.), Phys. Rev., 1953, 91, 1282.

[5] Newman (E.), Smith (A. M.) et Steigert (F. E.), Phys. Rev., 1961, 122, 1520.

[6] Schötт (W.), Z. Physik, 1964, 179, 428.

[7] Voltz (R.), Lopes da Silva (J.), Laustriat (G.) et Coche (A.), J. Chem. Phys., 1966, 45, 3306.

[8] Voltz (R.) et Laustriat (G.), J. Physique, 1968, 29, 159 .

[9] Voltz (R.), Dupont (H.) et Laustriat (G.), $J$. Physique, 1968, 29, 297.

[10] Lopes DA Silva (J.), Thèse Sc. Phys., Strasbourg, 1969.

[11] Kratochwill (F. I.), Z. Physik, 1970, 234, 74.
[12] Bönsch (G.) et Flammersfeld (A.), Z. Physik, 1970, 236, 305.

[13] Szalghary (W. D.), Z. Physik, 1970, 238, 130.

[14] Wille (K.), Z. Physik, 1971, 241, 55.

[15] Duquesne (M.) et Kaplan (J.), J. Physique Rad., 1960, 21, 708.

[16] Bollinger (L. M.) et Thomas (G. E.), Rev. Sci. Instr., 1961, 32, 1044.

[17] Pfeffer (G.), Thèse Sc. Phys., Strasbourg, 1965.

[18] Green (D. W.), J. Sci. Instr., 1961, 30, 833.

[19] Sternheimer (R. M.), Phys. Rev., 1952, 88, 851.

[20] NorthClifFe (L. C.), Ann. Rev. Nuclear Sci., 1963, $13,67$.

[21] Taylor (C. J.), Jentschke (W. K.), Remley (M. E.), Eby (F. S.) et Kruger (P. G.), Phys. Rev., 1951, 84, 1034.

[22] Fano (U.), Ann. Rev. Nuclear Sci., 1963, 13, 1.

[23] Bourland (P. D.) et Powers (D.), Phys. Rev. B, $1971,3,3635$. 